\title{
Interaction of Bordetella pertussis Virulence Components with Neutrophils: Effect on Chemiluminescence Induced by a Chemotactic Peptide and by Intact Bacteria
}

\author{
By F. F. CRAIG ${ }^{1,2 *}$ J. M. LACKIE, ${ }^{2}$ R. PARTON ${ }^{1}$ AND J. H. FREER ${ }^{1}$ \\ Departments of ${ }^{1}$ Microbiology and ${ }^{2}$ Cell Biology, University of Glasgow, Glasgow G12 8QQ, UK
}

(Received 14 December 1987; revised 21 March 1988)

The effect of secreted virulence components of Bordetella pertussis on chemiluminescence (CL) of rabbit peritoneal neutrophils was determined with the chemotactic peptide $N^{\prime}$-formyl-Lmethionyl-L-leucyl-L-phenylalanine (fMLP) or intact $B$. pertussis as the stimulus. Pertussis toxin (PT) inhibited the response to fMLP in a dose-dependent manner, although only after the neutrophils had been exposed to the toxin for $>15 \mathrm{~min}$. Both filamentous haemagglutinin (FHA) and lipopolysaccharide (LPS) markedly enhanced the CL response to fMLP after $\geqslant 15$ min incubation with the neutrophils. Similar effects to those of $B$. pertussis LPS were also seen with smooth and rough LPS from Salmonella minnesota. With the lowest dose of each component which elicited a maximal effect on CL, the inhibitory effect of PT overrode the enhancing effect of FHA and B. pertussis LPS. Pre-incubation of neutrophils with PT, FHA or $B$. pertussis LPS caused a slight reduction in the subsequent $C L$ response to virulent $B$. pertussis Tohama. Virulent (phase I, or X-mode) organisms of B. pertussis 18334 and B. pertussis Tohama induced greater neutrophil $C L$ than their avirulent ( $C$-mode) derivatives. There appeared to be an inverse correlation between bacterial hydrophilicity and the ability to induce neutrophil CL: $\mathrm{X}$-mode bacteria were significantly less hydrophilic than $\mathrm{C}$-mode organisms. Three mutants, the adenylate cyclase (AC)- and haemolysin (HLY)-deficient $B$. pertussis BP348, the FHA-deficient $B$. pertussis BP353, and the PT-deficient $B$. pertussis BP357, generated similar levels of CL and had similar hydrophilicity values. The hydrophilicity value of the avirulent mutant $B$. pertussis BP347 (deficient in AC, HLY, FHA and PT) and the CL induced by this strain were similar to those of $B$. pertussis $C$-mode organisms. Thus, the interaction of $B$. pertussis with neutrophils appears to be complex, reflecting both the alteration of leucocyte function by secreted virulence components of the organism and, in the absence of opsonins, the surface properties of the bacterium.

\section{INTRODUCTION}

Bordetella pertussis is the cause of pertussis (whooping cough), a disease of the respiratory tract (Manclark \& Cowell, 1984). Pertussis may be a severe disease in infants, and untreated cases are often complicated by secondary infections (Jamieson, 1973), suggesting that the normal defence mechanisms of the host are altered by $B$. pertussis infection. The lodgement and replication of $B$. pertussis in the respiratory tract would initiate an inflammatory response, with the subsequent recruitment of neutrophils to the infected area. Neutrophils play an important role in respiratory tract defence and patients with neutrophils which have chemotactic disorders are prone to recurrent respiratory illnesses (Smith et al., 1972; Gallin et al., 1980). The production of factors by $B$. pertussis which disrupt neutrophil function could therefore be important in pathogenesis of the disease and of related secondary infections.

Abbreviations: AC, adenylate cyclase; CL, chemiluminescence; FHA, filamentous haemagglutinin; fMLP, $N^{\prime}$-formyl-L-methionyl-L-leucyl-L-phenylalanine; HBS, HEPES-buffered saline; HLY, haemolysin, PT, pertussis toxin. 
Pertussis toxin (PT) is an important virulence component of $B$. pertussis (Weiss \& Hewlett, 1986) and elicits a wide variety of biological responses (Wardlaw \& Parton, 1983). It has profound effects on phagocyte function, including the inhibition of degranulation and chemotaxis of rabbit neutrophils in vitro induced by several chemotactic factors (Becker et al., 1985). Chemotactic factors are important in mediating inflammation and in the recruitment of neutrophils to a site of infection (Wilkinson, 1982). Migration and chemotaxis of murine macrophages are also suppressed by PT (Meade et al., 1984). Some of these effects on phagocytes are due to ADP-ribosylation of membrane-bound regulatory proteins by the toxin (Becker $e t$ al., 1986).

Other $B$. pertussis components implicated in virulence include adenylate cyclase (AC), filamentous haemagglutinin (FHA), and lipopolysaccharide (LPS). B. pertussis AC has a wide range of antiphagocytic properties (Confer \& Eaton, 1982) but unlike the other virulence factors may not be released into the growth medium (Hewlett \& Gordon, 1988). FHA is an adhesin of $B$. pertussis and its absence from the organism has been associated with loss of adherence to human ciliated respiratory-epithelial cells in vitro (Tuomanen \& Weiss, 1985). B. pertussis LPS has properties similar to those of other Gram-negative bacteria, such as pyrogenicity and the ability to promote non-specific immunity in mice (Nakase et al., 1970; Ayme et al., 1980).

The relationship between expression of virulence components by $B$. pertussis and its effect on neutrophil bactericidal responses is poorly understood. Light-production (chemiluminescence, CL) may be used as an indirect assay for neutrophil bactericidal responses and can be enhanced by 5-amino-2,3-dihydro-1,4-phthalazinedione (luminol), which is oxidized by products of the neutrophil myeloperoxidase system (DeChatelet et al., 1982; Dahlgren \& Stendahl, 1983). The aim of the present investigation was to determine the effects of secreted virulence components of $B$. pertussis on luminol-enhanced CL of neutrophils induced by a soluble (the chemotactic factor $N^{\prime}$-formyl-L-methionyl-L-leucyl-L-phenylalanine; fMLP) and by a particulate (intact bacteria) stimulus. Strains of $B$. pertussis that are altered in a range of virulence components have been derived by transposon-insertion mutagenesis (Weiss et al., 1983) or can be produced by modifying the growth medium to yield either virulent (phase I, or X-mode) or avirulent (Cmode) organisms (Lacey, 1960; Robinson et al., 1986). The luminol-enhanced CL response of neutrophils to these different strains was also determined. Rabbit neutrophils were used for our study since the rabbit is susceptible to $B$. pertussis aerosol infection and therefore constitutes a relevant model for the disease in humans (Preston et al., 1980; Ashworth et al., 1982).

\section{METHODS}

Bacteria and growth conditions. The $B$. pertussis strains used and their relevant properties are listed in Table 1 . Strains Tohama and 18334 were from departmental stock cultures. The other four strains were kindly supplied by Dr A. A. Weiss (Department of Microbiology and Immunology, Box 678, Medical College of Virginia, Richmond, Virginia 23298-0001, USA) and were derived from B. pertussis strain Tohama as described by Weiss et al. (1983).

A loopful of growth from a $72 \mathrm{~h}$ culture grown at $37^{\circ} \mathrm{C}$ on Bordet-Gengou medium, containing $20 \%(\mathrm{v} / \mathrm{v})$ sterile, defibrinated horse blood, was used to inoculate each litre of Stainer-Scholte medium (Stainer \& Scholte, 1971) modified such that the Tris concentration was $1.25 \mathrm{~g}^{-1}$. Bacteria were grown either in $\mathrm{X}$-mode $(\mathrm{NaCl}$ containing) medium or in $\mathrm{C}$-mode medium (containing $\mathrm{MgSO}_{4}$ instead of $\mathrm{NaCl}$ ) for $48 \mathrm{~h}$ at $37^{\circ} \mathrm{C}$ in an orbital incubator at 80 r.p.m. (Brownlie et al., 1985). Cultures were centrifuged $\left(6000 \mathrm{~g}\right.$ for $30 \mathrm{~min}$ at $4{ }^{\circ} \mathrm{C}$ ) and the sedimented bacteria stored at $-70^{\circ} \mathrm{C}$ in $9.6 \mathrm{mM}$-phosphate-buffered saline (PBS; $0.285 \mathrm{M}-\mathrm{NaCl}, 0.006 \mathrm{M}-\mathrm{KCl}$, $0.016 \mathrm{M}-\mathrm{Na}_{2} \mathrm{HPO}_{4}, 0.003 \mathrm{M}-\mathrm{KH}_{2} \mathrm{PO}_{4}, \mathrm{pH} 7 \cdot 3$ ) containing $10 \%$ (v/v) glycerol until used.

Reagents. Bordet-Gengou medium and defibrinated horse blood were both obtained from Gibco. PT (kindly supplied by Dr V. Y. Perera, Department of Microbiology, Glasgow University), was purified by the method of Perera et al. (1985). Briefly, the toxin was isolated by fetuin-Sepharose affinity chromatography of cytoplasmic fractions of $B$. pertussis 18334, phase I, grown for $48 \mathrm{~h}$ at $37^{\circ} \mathrm{C}$ in cyclodextrin liquid medium (Imaizumi et al., 1983). No detectable $A C$ activity was found in a sample ( $10 \mu \mathrm{g}$ protein per assay tube) of the purified toxin. The AC assay was performed as described by Brownlie et al. (1985) except that calmodulin (bovine brain) was added to the reaction mixture to a final concentration of $0.3 \mu \mathrm{M}$ to increase sensitivity. The assay could detect a minimum of 0.2 pM-cAMP per assay tube. FHA was kindly provided by Dr L. A. E. Ashworth (Public Health Laboratory Service Centre for Applied Microbiology and Research, Porton Down, Wilts SP4 0JG, UK). LPS from B. pertussis 165 (phase I) was obtained from LIST Biological Laboratories. Salmonella minnesota (smooth) LPS, S. minnesota 
Table 1. B. pertussis strains used

Organism (X-mode)

Virulence component(s) absent

B. pertussis 18334

B. pertussis Tohama

None

B. pertussis BP347

None

AC, FHA, PT, haemolysin (HLY),

heat-labile toxin

B. pertussis BP348

$B$. pertussis BP353

B. pertussis BP357

AC, HLY

FHA

PT*

* Some cell-associated PT present (Weiss et al., 1983).

Re595 (rough) LPS, calmodulin, EDTA, oyster glycogen, HEPES, luminol and fMLP were all obtained from Sigma. All LPS preparations were reconstituted according to the supplier's instructions except that HEPESbuffered saline (HBS) was used in place of distilled water. Normal HBS contained $\left(\mathrm{g} \mathrm{l}^{-1}\right): \mathrm{NaCl}, 8 ; \mathrm{KCl}, 0.4$; $\mathrm{MgCl}_{2} \cdot 6 \mathrm{H}_{2} \mathrm{O}, 0 \cdot 2 ; \mathrm{CaCl}_{2}, 0 \cdot 14$; glucose, 1 ; and HEPES, $2 \cdot 388$. Divalent-cation-free HBS-EDTA was prepared by the omission of calcium and magnesium salts and addition of $0.292 \mathrm{~g} \mathrm{EDTA}^{-1}$. Both solutions were adjusted to pH 7.4 using $1 \mathrm{M}-\mathrm{NaOH}$.

Hydrophilicity assay. Hydrophilicity of intact bacteria was measured by a modification of the method of Robinson et al. (1983). Bacterial suspensions were washed twice in PBS by centrifugation ( $9000 \mathrm{~g}$ for $1 \mathrm{~min}$ ) and resuspended in PBS to an OD $_{550}$ of $1 \cdot 00$. $N$-Octane $(300 \mu \mathrm{l})$ was added to $4 \mathrm{ml}$ of the bacterial suspension and the suspension vortex-mixed for $1 \mathrm{~min}$. After standing for $20 \mathrm{~min}$ at room temperature, the upper, hydrophobic layer was removed and the $\mathrm{OD}_{550}$ of the remaining aqueous layer determined. This value, after correction for a PBS control, was multiplied by 100 and expressed as the percentage hydrophilicity of the organism.

Neutrophil preparation. Peritoneal neutrophils were obtained from healthy female New Zealand White rabbits with no history of Bordetella bronchiseptica infection. Briefly, $500 \mathrm{ml} 0 \cdot 85 \%(\mathrm{w} / \mathrm{v}) \mathrm{NaCl}$ containing $0 \cdot 1 \%(\mathrm{w} / \mathrm{v})$ oyster glycogen was injected intraperitoneally and the peritoneal exudate collected $4 \mathrm{~h}$ later (Lackie, 1977). The exudate was stored at $4{ }^{\circ} \mathrm{C}$ and used within $2 \mathrm{~d}$ of isolation. Before use the cells were washed once in divalentcation-free HBS-EDTA by centrifugation $\left(700 \mathrm{~g}\right.$ for $60 \mathrm{~s}$ at $\left.4^{\circ} \mathrm{C}\right)$. Contaminating erythrocytes were then removed from the cell pellet by resuspension in $2 \mathrm{ml}$ distilled water for $5 \mathrm{~s}$, restoration of isotonicity with HBS, and recentrifugation of the cell suspension. Washed cells were used within $2 \mathrm{~h}$ of preparation. To obtain a monodisperse population of cells for the assay the neutrophils were passed through a $10 \mu \mathrm{m}$ pore size Nitex filter (Plastok Associates). Cell suspensions contained $>95 \%$ neutrophils as assessed by microscopy and cell viability was $>95 \%$ as determined by Trypan Blue exclusion. Cell numbers were standardized by counting with a haemocytometer.

$C L$ assay. This assay was performed using a Wallac LKB 1251 luminometer connected to an Acorn BBC B microcomputer. $\mathrm{CL}$ emission was measured in $\mathrm{mV}$ at $37^{\circ} \mathrm{C}$. The number of neutrophils per assay tube was $10^{6}$ in a final volume of $700 \mu \mathrm{l}$. The luminometer was run in continuous mixing mode throughout all assays, each sample being assayed in duplicate or triplicate. When fMLP was the stimulus, samples were counted for $0.5 \mathrm{~s}$ every $90 \mathrm{~s}$ over a period of approximately $30 \mathrm{~min}$, which gave 20 counts per sample. When bacteria were the stimulus, samples were counted for $12 \mathrm{~s}$ every $225 \mathrm{~s}$ over a period of approximately $60 \mathrm{~min}$, which gave 17 counts per sample. Thus, although the CL curves are shown as continuous traces, they are derived from a finite number of observations ( 17 or 20 depending on the stimulus), each observation being the mean of replicate samples. In some cases the peak values and total CL counts for each sample were tabulated for ease of comparison. The peak value was the highest $C L$ value obtained over the period of the assay while total CL was the sum of the individual CL counts over the period of the assay. The final concentration of luminol in all assays was $10 \mu \mathrm{M}$. When fMLP was the stimulus, both fMLP and luminol were added as a mixture at the start of each assay by automated dispenser. The final concentration of $\mathrm{MLP}$ in the assays was $0.1 \mu \mathrm{M}$. Bacterial suspensions used as stimuli were first washed twice in $\mathrm{HBS}$ then standardized to an $\mathrm{OD}_{600}$ of 0.5 (equivalent to a bacterial dry weight of $255 \mu \mathrm{g} \mathrm{ml}^{-1}$ ). A sample $(50 \mu 1)$ of this suspension constituted the bacterial stimulus and was added to pre-heated $\left(20 \mathrm{~min}\right.$ at $\left.37^{\circ} \mathrm{C}\right)$ neutrophils prior to automated dispensing of luminol. The ratio of bacteria to neutrophils in the assay was approximately $15: 1$.

For pre-treatment with a bacterial component(s), neutrophils were pre-incubated in the presence of the component(s) in the luminometer at $37^{\circ} \mathrm{C}$ for $60 \mathrm{~min}$, unless otherwise stated. The assay was then started by addition of the stimulus and luminol and counting of the samples.

The Student $t$-test or the Fisher-Behrens test was used to evaluate the significance of differences between the two population means in various experiments (Campbell, 1981). 


\section{RESULTS}

\section{Effect of individual virulence components on neutrophil $C L$ response to fMLP}

Human peripheral neutrophils gave a bimodal response in the presence of IMLP and luminol (Bender \& Van Epps, 1983). A similar bimodal response was observed with rabbit peritoneal neutrophils (Fig. 1). In these cells, pre-incubation with PT for $60 \mathrm{~min}$ caused a dose-dependent inhibition of the CL induced by fMLP : $6 \mathrm{ng}$ caused a slight suppression and $160 \mathrm{ng}$ an almost complete suppression of $\mathrm{CL}$ with $10^{6}$ cells. However, similar pre-incubation with the toxin had no effect on luminol-enhanced CL of neutrophils in the absence of fMLP (Table 2).

Pre-treatment with FHA for 60 min enhanced the CL of neutrophils induced by FMLP at doses of $10-50 \mathrm{ng}$ per $10^{6}$ cells (Fig. $2 a$ ). A dose of $250 \mathrm{ng}$ had approximately the same enhancing effect as a dose of $50 \mathrm{ng}$ over the first $10 \mathrm{~min}$ of the assay, although a slight inhibitory effect was observed after $20 \mathrm{~min}$ (data not shown). Similar pre-treatment with a dose of $50 \mathrm{ng}$ of FHA slightly stimulated luminol-enhanced CL even in the absence of fMLP (Table 2).

Pre-incubation with $B$. pertussis LPS for 60 min enhanced neutrophil CL induced by fMLP at doses $\geq 1 \mu \mathrm{g}$ per $10^{6}$ cells (Fig. $2 b$ ). Maximal enhancement occurred with a dose of $10 \mu \mathrm{g}$. Although increased neutrophil CL was obtained with doses of LPS exceeding $10 \mu \mathrm{g}$, cell viability was diminished by such treatment. As with FHA, pre-treatment with $B$. pertussis LPS for 60 min slightly enhanced neutrophil CL in the absence of fMLP (Table 2).

$B$. pertussis LPS is a rough LPS (Peppler, 1984) and was compared to the smooth LPS from Salmonella minnesota and the rough LPS from $S$. minnesota Re595 to determine if LPS enhancement of neutrophil CL was specific to B. pertussis LPS, or typical of LPSs in general. After pre-treatment of neutrophils with LPS for $60 \mathrm{~min}, S$. minnesota rough LPS was the most active, $B$. pertussis the second most active, while $S$. minnesota smooth LPS was the least active enhancer of neutrophil CL induced by fMLP (Table 3). The higher dose $(10 \mu \mathrm{g})$ of $S$. minnesota smooth LPS was not as active as the lower dose $(1 \mu \mathrm{g})$, suggesting a slight degree of toxicity at the higher dose. Pre-incubation with any of the three LPS types stimulated neutrophil CL in the absence of fMLP (Table 2).

\section{Influence of pre-incubation time on activity of the virulence components}

The time of pre-incubation of neutrophils with individual virulence components of $B$. pertussis profoundly affected the subsequent CL response to fMLP (Fig. 3). Neutrophils had to be exposed to PT for $>15$ min before any inhibitory effects were observed. An enhanced CL response following exposure to FHA or LPS was first detectable $15 \mathrm{~min}$ after addition of these agents to the neutrophils.

\section{Effect of mixtures of virulence components on neutrophil $C L$ response to fMLP}

PT inhibited fMLP-induced CL of rabbit peritoneal neutrophils, whereas FHA enhanced it. Since both factors are released during bacterial growth, it was of interest to determine which effect would predominate. After pre-incubation for $60 \mathrm{~min}$ with equal doses $(50 \mathrm{ng})$ of both factors, the suppressive effect of PT overrode the enhancing effect of FHA (Table 4). Preincubation of neutrophils with $50 \mathrm{ng}$ of FHA and a higher dose of PT (160 ng) caused a further reduction in CL. Similarly, with $10 \mu \mathrm{g}$ of $B$. pertussis LPS and an inhibitory dose of PT (160 ng), the inhibitory activity of PT almost completely suppressed the enhancing effect of LPS (Table 4).

\section{Effect of purified virulence components on neutrophil $C L$ response to virulent $B$. pertussis}

To further determine the influence of secreted virulence components of $B$. pertussis on neutrophil responses, $C L$ studies were performed using $B$. pertussis Tohama $X$-mode as the stimulus (Fig. 4). The CL peak values occurred within the first $60 \mathrm{~min}$ of the assay, although the responses took up to $2 \mathrm{~h}$ to decline to background levels. Most experiments were run for $60 \mathrm{~min}$, which was sufficient to detect any differences in the $C L$ patterns generated. Pre-treatment of neutrophils with $160 \mathrm{ng}$ PT for $60 \mathrm{~min}$ caused about $30 \%$ inhibition $(P<0.05)$ of the peak value and total CL induced by the bacterial stimulus. Pre-treatment for 60 min with either $50 \mathrm{ng}$ FHA or $10 \mu \mathrm{g}$ LPS induced an initial increase in the rate of CL generation followed by a decrease, the overall effect being a slight reduction in the peak value and total $\mathrm{CL}$ production $(P<0.05)$. 


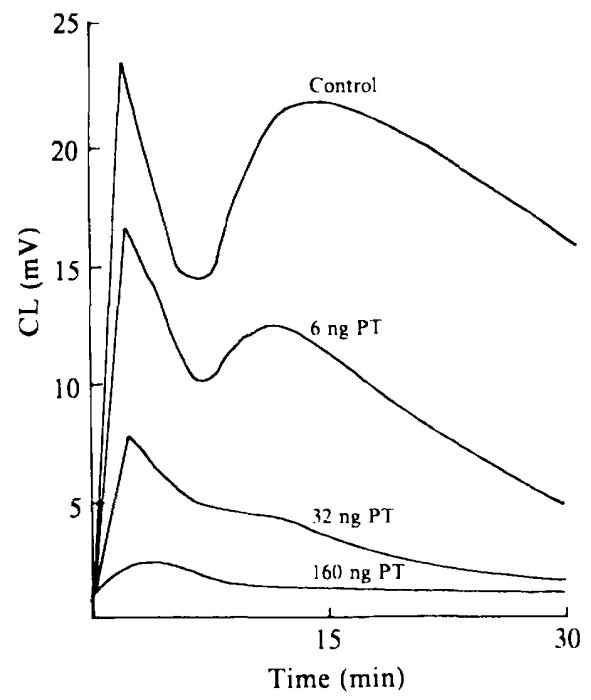

Fig. 1
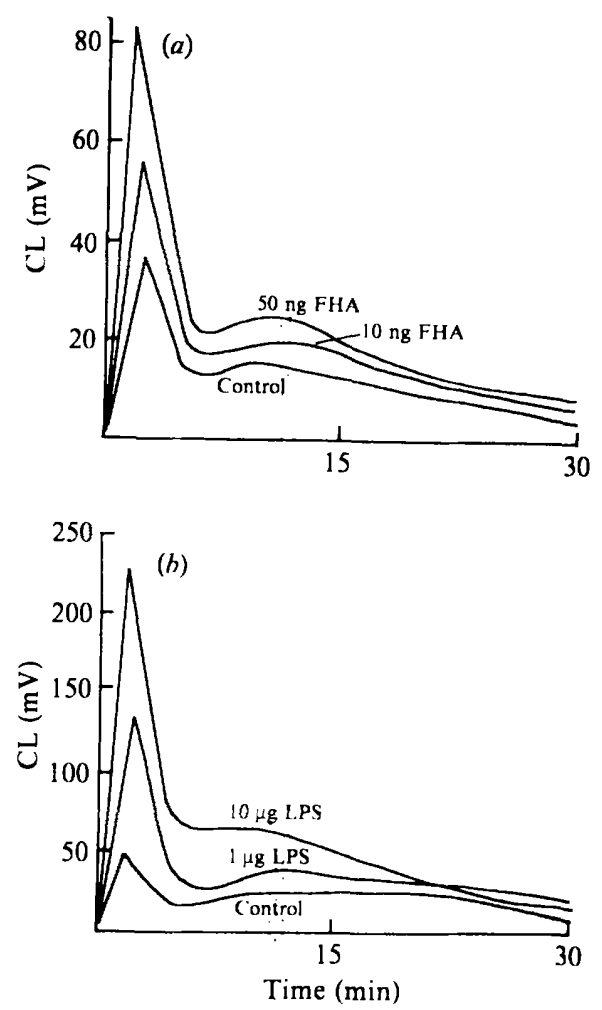

Fig. 2

Fig. 1. Inhibition of fMLP-induced CL of neutrophils by PT. The figure shown is a mean of triplicate observations from one assay and is representative of seven separate experiments. The highest SEM value recorded was \pm 0.6 for the first peak and \pm 0.5 for the second peak.

Fig. 2. Enhancement of fMLP-induced CL of neutrophils by (a) FHA and $(b) B$. pertussis LPS. The figures shown are a mean of triplicate observations from one assay and are representative of four separate experiments. The highest SEM value noted was \pm 4 for the first peak and \pm 2 for the second peak.

Table 2. Stimulation of neutrophil $C L$ by purified virulence components in the absence of $f M L P$

$\begin{array}{lrrr}\begin{array}{c}\text { Test } \\ \text { substance }\end{array} & \begin{array}{c}\text { Dose per } \\ \text { assay } \\ \text { mixture }\end{array} & \overbrace{\text { Experimental }}^{\text {CL }(\mathrm{mV}) \pm \text { SEM } \dagger} & \text { Control } \\ \text { PT } & 160 \mathrm{ng} & 18 \pm 1 & 18 \pm 1 \\ \text { FHA } & 50 \mathrm{ng} & 21 \pm 1 * * & 18 \pm 1 \\ B . \text { pertussis } \text { LPS } & 1 \mu \mathrm{g} & 26 \pm 1 * * & 19 \pm 1 \\ S . \text { minnesota } \text { smooth LPS } & 1 \mu \mathrm{g} & 24 \pm 1 * * & 19 \pm 1 \\ S . \text { minnesota } \text { rough LPS } & 1 \mu \mathrm{g} & 62 \pm 1 * * & 19 \pm 1\end{array}$

+ After pre-incubation, individual CL counts were summed over $30 \mathrm{~min}$ and the mean value obtained for each sample $(n>4$ observations). For experimental versus control values: $* * *=P<0.001, * *=P<0.01$, $*=P<0.05$, otherwise not significantly different from control value $(P>0.05)$.

Pre-incubation of neutrophils with a mixture of PT (50 ng) and FHA (50 ng) for $60 \mathrm{~min}$ reduced both the subsequent $C L$ peak value and total $C L$ response to $B$. pertussis Tohama, and the extent of reduction appeared to be a cumulative effect of PT and FHA alone (Table 4).

\section{Neutrophil $C L$ induced by different strains of $B$. pertussis}

The effect on neutrophil CL of the loss of a range of cell-associated virulence components by the process of antigenic modulation of two B. pertussis strains, 18334 and Tohama, was 


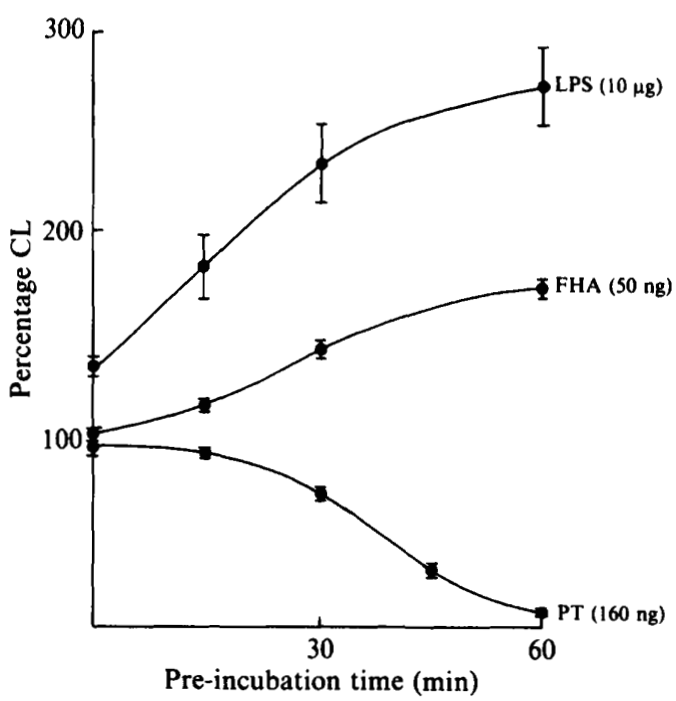

Fig. 3. Influence of pre-incubation time on the effect of $B$. pertussis virulence components on neutrophil CL induced by $\mathrm{fMLP}$. The individual CL counts were summed over $30 \mathrm{~min}$ and expressed as percentage $C L$ relative to the control $(=100 \%)$. Each point shown is the mean of six observations obtained from duplicate samples in three separate experiments; bars represent SEM.

Table 3. Influence of $B$. pertussis and S. minnesota LPSs on neutrophil $C L$ induced by $f M L P$

$$
\begin{gathered}
\text { Test } \\
\text { substance }
\end{gathered}
$$

\section{Control}

B. pertussis LPS

S. minnesota smooth LPS

S. minnesota rough LPS

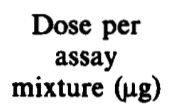

mixture $(\mu \mathrm{g})$

$\overbrace{\text { Peak value }}^{\text {CL }(\mathrm{mV}) \pm \text { SEM } \dagger}$

† Derived as in Table $2 ; n=6$ observations.

Table 4. Influence of mixtures of $B$. pertussis virulence components on neutrophil $C L$ induced by

\begin{tabular}{|c|c|c|c|}
\hline \multirow[b]{2}{*}{ Stimulus } & \multirow{2}{*}{$\begin{array}{l}\text { Test substance } \\
\text { (dose per assay } \\
\text { mixture) }\end{array}$} & \multicolumn{2}{|c|}{$\mathrm{CL}(\mathrm{mV}) \pm \mathrm{SEM} \dagger$} \\
\hline & & Peak value & Total \\
\hline fMLP & 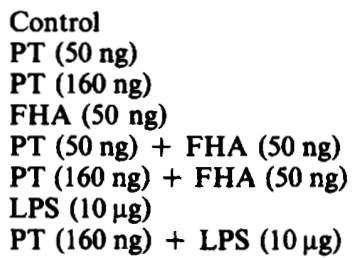 & $\begin{aligned} 20 & \pm 1 \\
7 & \pm 2 * * \\
3 & \pm 1 * * \\
117 & \pm 16 * * \\
14 & \pm 4 \\
7 & \pm 1 * * * \\
108 & \pm 3 * * \\
5 & \pm 1 * * *\end{aligned}$ & $\begin{aligned} 292 & \pm 16 \\
83 & \pm 23 * * * \\
40 & \pm 9 * * \\
868 & \pm 73 * * \\
142 & \pm 42 * \\
65 & \pm 12 * * * \\
898 & \pm 14 * * * \\
51 & \pm 2 * * *\end{aligned}$ \\
\hline B. pertussis & $\begin{array}{l}\text { Control } \\
\text { PT (50 ng) } \\
\text { FHA (50 ng) } \\
\text { PT (50 ng) + FHA (50 ng) }\end{array}$ & $\begin{array}{l}333 \pm 14 \\
276 \pm 14 * \\
292 \pm 7 * \\
241 \pm 9 * * *\end{array}$ & $\begin{array}{l}4338 \pm 154 \\
3654 \pm 166 * * \\
3851 \pm 93 * \\
3085 \pm 104 * * *\end{array}$ \\
\hline
\end{tabular}
fMLP or by $B$. pertussis Tohama $X$-mode organisms

† Derived as in Table 2; $n=9$ observations. 

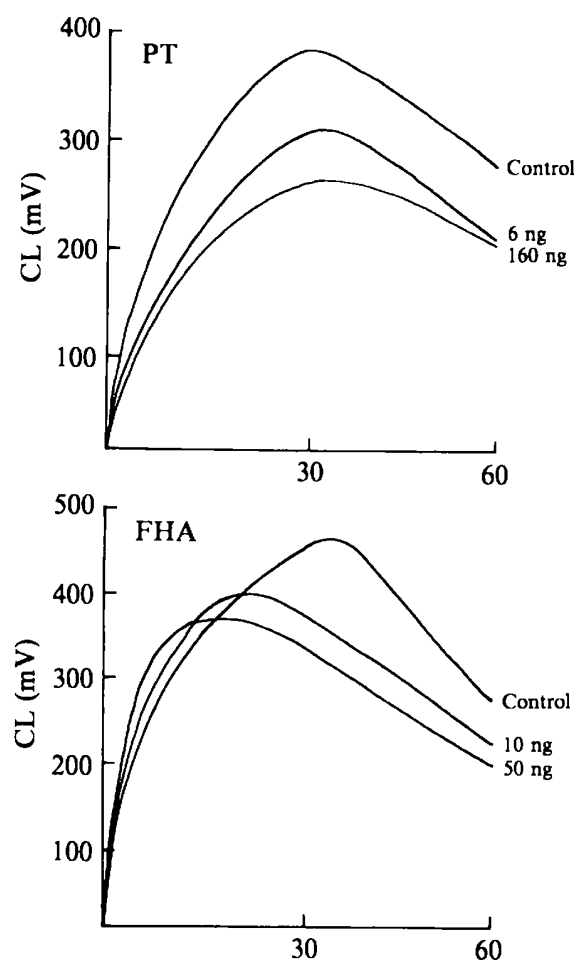

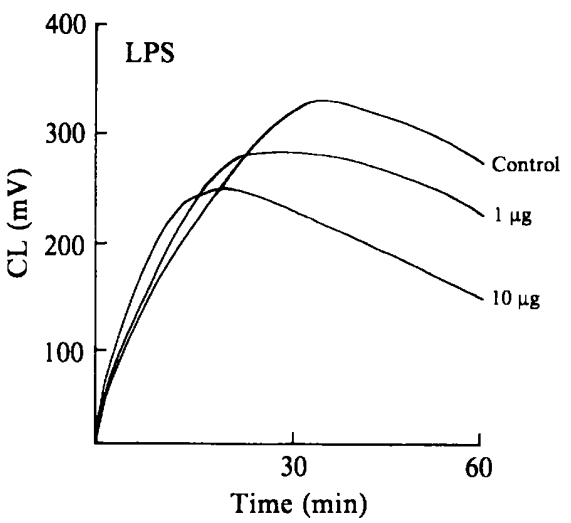

Fig. 4

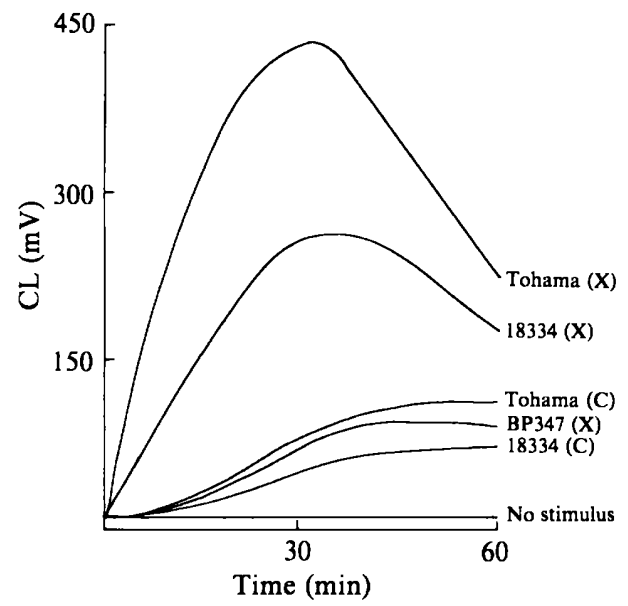

Fig. 5

Fig. 4. Effect of purified virulence components of $B$. pertussis on generation of neutrophil $C L$ to $B$. pertussis Tohama $X$-mode organisms. Each figure shown is the mean of triplicate observations from one assay and is representative of at least four separate experiments. The highest SEM value recorded was \pm 25 .

Fig. 5. Stimulation of neutrophil CL by various strains of $B$. pertussis. The mode of the organism is given in parentheses. The figure shown is the mean of eight observations obtained from duplicate samples in four separate experiments. The highest SEM value observed was \pm 14 . The overall $C L$ responses stimulated by $B$. pertussis Tohama (C), B. pertussis BP347 (X) and B. pertussis $18334(C)$ were not significantly different $(P>0.05)$.

investigated. $B$. pertussis Tohama and $18334 \mathrm{C}$-mode organisms induced lower CL peaks and lower total levels of $\mathrm{CL}$ than their respective virulent $\mathrm{X}$-mode counterparts (Fig. 5). The $B$. pertussis Tohama $\mathrm{X}$-mode strain induced a significantly higher CL peak value and higher total $C L$ than the $B$. pertussis $18334 \mathrm{X}$-mode strain $(P<0.001)$, perhaps reflecting inter-strain differences in expression of biologically active products. A transposon-induced avirulent 


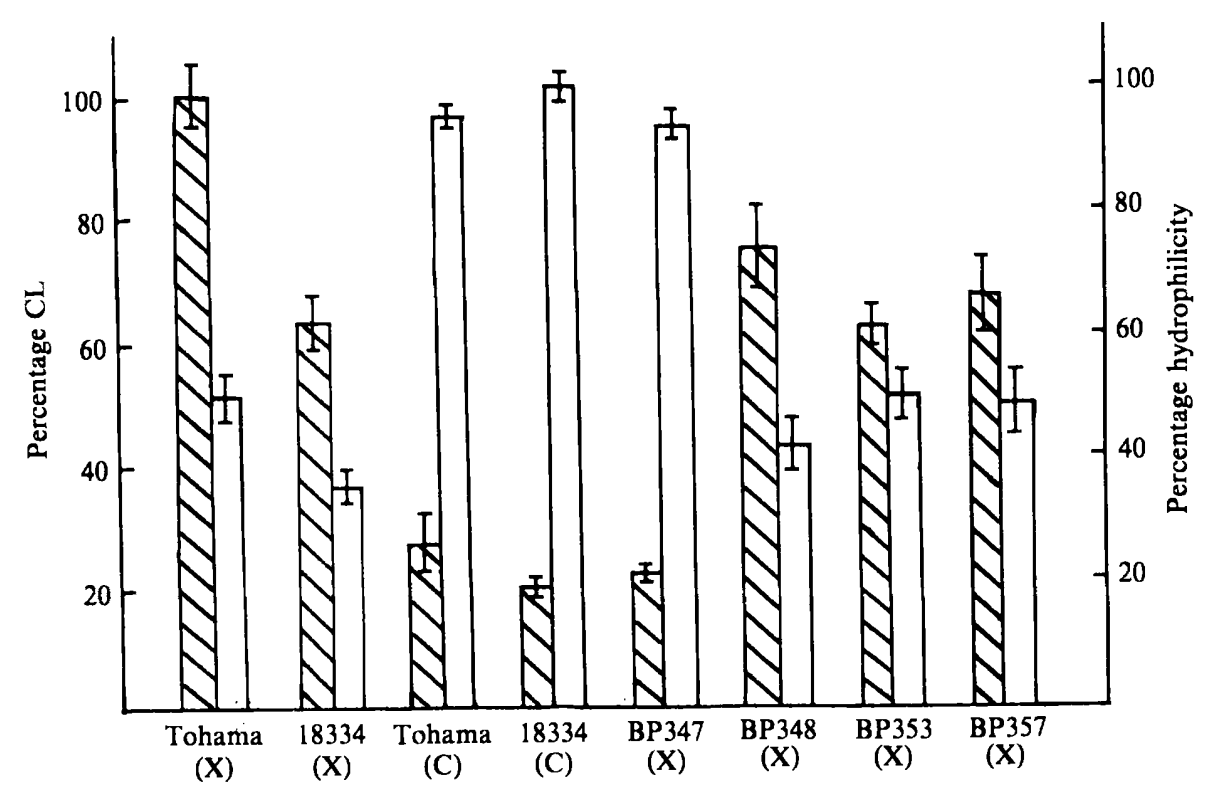

Fig. 6. A comparison of hydrophilicity (open bars) of strains of $B$. pertussis with their generation of neutrophil $\mathrm{CL}$ (hatched bars). The $\mathrm{CL}$ results are the total counts over $60 \mathrm{~min}$. Percentage $\mathrm{CL}$ was expressed relative to the level of $\mathrm{CL}$ induced by the $B$. pertussis Tohama (X) strain (taken as $100 \%$ ). Each point shown is the mean of between 8 and 12 observations obtained from duplicate samples in four to six separate experiments; bars represent SEM.

mutant, B. pertussis BP347, grown in X-mode culture medium, induced a similar CL pattern to the $\mathrm{C}$-mode organisms.

To assess the role of individual, cell-associated, virulence components of $\boldsymbol{B}$. pertussis on the neutrophil $\mathrm{CL}$ response, several transposon-induced mutants deficient in either one or two virulence components were assayed and compared with the CL response to virulent $B$. pertussis. The total CL generated by all strains correlated well with the CL peak values, and hence only total CL values are shown for these strains (Fig. 6). The CL responses generated by the AC- and HLY-deficient mutant B. pertussis BP348, the FHA-deficient mutant B. pertussis BP353, and the PT-deficient mutant $B$. pertussis BP357 were not significantly different $(P>0.05)$. Although reduced compared to Tohama X-mode organisms $(P<0.01)$, the CL stimulated by these three mutants was still higher than that generated in response to any of the $\mathrm{C}$-mode bacteria and was similar to that to $18334 \mathrm{X}$-mode organisms.

\section{Hydrophilicity of the B. pertussis strains}

The hydrophilicity of a bacterium is important in determining its interaction with other cells, and so the hydrophilicity values of the $B$. pertussis strains used in the CL studies were measured. $B$. pertussis BP347 and the $B$. pertussis C-mode organisms were significantly more hydrophilic $(P<0.01)$ than $B$. pertussis X-mode organisms (Fig. 6). B. pertussis BP347 was the only strain to differ significantly $(P<0.01)$ in hydrophilicity when compared to other $\mathrm{X}$-mode strains.

\section{DISCUSSION}

A significant observation in this study was that FHA and LPS enhanced, whereas PT inhibited, neutrophil CL induced by fMLP. In addition, PT overrode the enhancing effect of FHA or B. pertussis LPS. PT inhibits both rabbit and human neutrophil chemotaxis and degranulation induced by fMLP (Becker et al., 1985; Lad et al., 1985). Our results suggest that neutrophil myeloperoxidase-associated responses to fMLP would also be suppressed during a 
pertussis infection, even in the presence of stimulatory components such as FHA or $B$. pertussis LPS. The suppression of neutrophil responses to chemotactic factors by PT could inhibit stimulation of bactericidal activity and leucocyte recruitment to an area of infection. This inhibition may prolong survival of $B$. pertussis or secondary invaders in vivo.

The stimulation of neutrophil CL by $B$. pertussis LPS, in the absence or presence of fMLP, was non-specific since both $S$. minnesota smooth and rough LPS had similar effects. There was an association between roughness of LPS and stimulation of neutrophil CL. Similar results have been reported with smooth and rough Escherichia coli LPS and human neutrophils in the absence of fMLP (Henricks et al., 1983).

The susceptibility of a bacterium to interaction with phagocytes decreases as bacterial hydrophilicity increases (Stendahl, 1983). In agreement with earlier studies (Robinson et al., 1983), we found that $B$. pertussis $\mathbf{C}$-mode strains were more hydrophilic than their $\mathrm{X}$-mode counterparts. There appeared to be an inverse correlation between hydrophilicity and neutrophil CL induced by $B$. pertussis $\mathrm{X}$ - and $\mathrm{C}$-mode organisms. An association between bacterial hydrophilicity and induction of poor CL by human neutrophils with unopsonized Escherichia coli was reported by Topley et al. (1984). Since CL is an indicator of neutrophil bactericidal activity (Horan et al., 1982), B. pertussis C-mode bacteria seem to stimulate rabbit neutrophils less than their respective X-mode strains, but it is not known if this is the case with $B$. pertussis strains and human neutrophils.

The transposon-induced avirulent mutant $B$. pertussis BP347 (Weiss et al., 1983) is similar to $\mathrm{C}$-mode organisms in that both are deficient in a number of virulence components (Robinson $e$ al., 1986). B. pertussis BP347 was similar to C-mode organisms in being more hydrophilic than $\mathrm{X}$-mode organisms and in stimulating a low level of neutrophil $\mathrm{CL}$. Three other transposoninduced mutants, $B$. pertussis BP348, B. pertussis BP353 and B. pertussis BP357, are deficient in only one or two virulence components. These three mutants had similar hydrophilicity values and induced levels of CL comparable to those of $X$-mode organisms. Thus, the transition of $B$. pertussis from the hydrophobic to hydrophilic state is not solely due to loss in AC and HLY, FHA or PT.

Although CL was similar with virulent $B$. pertussis 18334 and FHA- or PT-deficient mutants as stimulus, neutrophils pre-treated with exogenous FHA or PT gave slightly suppressed CL responses to virulent $B$. pertussis. With the ratio of bacteria to neutrophils used there may not have been enough of each virulence factor on the surface of the washed bacterial cells to cause inhibition of CL. Also this may explain the discrepancy between reports of purified AC inhibiting neutrophil CL induced by bacteria (Friedman et al., 1987) and the present study showing that an AC- and HLY-deficient mutant of $B$. pertussis induced less CL than a strain which produced both components.

The data of Friedman et al. (1987) showed that PT has only a slight inhibitory effect on the CL of human neutrophils induced by Staphylococcus aureus. We found a similar pattern with rabbit neutrophils and $B$. pertussis. In addition, PT has only a slight inhibitory effect on neutrophil release of myeloperoxidase to another particulate stimulus, opsonized zymosan (Spangrude $e t$ al., 1985). Thus, the interaction of particulate stimuli with neutrophils is more resistant to disruption by PT than are responses stimulated by chemotactic factors. This suggests that the PT-sensitive regulatory protein involved in stimulation by chemotactic factors is not so important in mediating the neutrophil response to particulate stimuli.

FHA and $B$. pertussis LPS had different effects on the overall response of neutrophils to fMLP and to $B$. pertussis. However, both components had an initial stimulatory effect, over about the first 15 min of each assay, with either stimulus. The influence of these components on neutrophil responses may depend on the nature of the stimulus and on the time-course over which the stimulus has its major effect. To our knowledge this is the first report of FHA showing biological activity with phagocytes. The effects of FHA on neutrophil CL responses suggest that this factor may also influence other neutrophil functions. FHA is a major adhesin of $B$. pertussis (Manclark \& Cowell, 1984; Tuomanen \& Weiss, 1985), and like other bacterial adhesins (Scheffer et al., 1985), it could play a role in pathogenesis by induction of the release of inflammatory mediators from neutrophils. 
In conclusion, exogenous FHA and LPS enhanced fMLP-induced CL of neutrophils but had an overall slight inhibitory effect on the neutrophil response to virulent $B$. pertussis. However, the inhibitory effect of PT overrode the enhancing effect of both FHA and $B$. pertussis LPS on the neutrophil response to $\mathrm{FLP}$, suggesting that inhibition of CL could provide a sensitive, in vitro assay for PT (Craig et al., 1988). The neutrophil CL pattern induced by an individual strain of unopsonized $B$. pertussis appears to be influenced by strain hydrophilicity. Although unopsonized $B$. pertussis $C$-mode organisms are more resistant to interaction with neutrophils than are X-mode organisms, the production of virulence factors, such as PT and AC (Weiss \& Hewlett, 1986), by X-mode bacteria would probably suppress neutrophil bactericidal responses in vivo and therefore prolong the survival of the bacterium or secondary pathogens. Thus, the interaction of $B$. pertussis with neutrophils appears to be complicated, depending on disruption of leucocyte function by secreted virulence components and, in the absence of opsonins, on surface components of the organism.

This work was funded by grant no. HERT 654 from the Scottish Hospitals Endowments Research Trust.

\section{REFERENCES}

Ashworth, L. A. E., Fitzgeorge, R. B., Irons, L. I., MORGaN, C. P. \& Robinson, A. (1982). Rabbit nasopharyngeal colonization by Bordetella pertussis: the effects of immunization on clearance and on serum and nasal antibody levels. Journal of Hygiene 88, 475-486.

Ayme, G., Caroff, M., Chaby, R., HaeffnerCavaillon, N., Le Dur, A., Moreau, M., Muset, M., Mynard, M.-C., Roumiantzeff, M., Schulz, D. \& SZABO, L. (1980). Biological activities of fragments derived from Bordetella pertussis endotoxin: isolation of a non-toxic, Schwartzman-negative lipid A possessing high adjuvant properties. Infection and Immunity 27, 739-745.

Becker, E. L., Kermode, J. C., Naccache, P. H., Yassin, R., MARSh, M. L., Munoz, J. J. \& Sha'Afi, R. 1. (1985). The inhibition of neutrophil granule enzyme secretion and chemotaxis by pertussis toxin. Journal of Cell Biology 100, 1641-1646.

Becker, E. L., Kermode, J. C., Naccache, P. H., Yassin, R., Munoz, J. J., Marsh, M. L., Huang, C.-K. \& SHA'AFI, R. I. (1986). Pertussis toxin as a probe of neutrophil activation. Federation Proceedings 45, 2151-2155.

BENDER, J. G. \& VAN EPPS, D. E. (1983). Analysis of the bimodal chemiluminescence pattern stimulated in human neutrophils by chemotactic factors. Infection and Immunity 41, 1062-1070.

Brownlie, R. M., PARToN, R. \& CoOTE, J. G. (1985). The effect of growth conditions on adenylate cyclase activity and virulence-related properties of Bordetella pertussis. Journal of General Microbiology 131, 17-25.

CAMpbell, R. C. (1981). Statistics for Biologists. Cambridge: Cambridge University Press.

CONFER, D. L. \& EATON, J. W. (1982). Phagocyte impotence caused by an invasive bacterial adenylate cyclase. Science 217, 948-950.

Craig, F. F., Christodoulides, M., Parton, R., Stewart-Tull, D. E. S., Freer, J. H. \& LaCKIE, J. M. (1988). A sensitive, chemiluminescence assay for pertussis toxin and for evaluation of cell-free pertussis toxoids. FEMS Microbiology Immunology (in the Press).
DAHLgRen, C. \& Stendahl, O. (1983). Role of myeloperoxidase in luminol-dependent chemiluminescence of polymorphonuclear leukocytes. Infection and Immunity 39, 736-741.

DeChatelet, L. R., Long, G. D., Shirley, P. S., Bass, D. A., Thomas, M. J., Henderson, F. W. \& Cohen, M. S. (1982). Mechanism of the luminol-dependent chemiluminescence of human neutrophils. Journal of Immunology 129, 1589-1593.

Friedman, R. L., Fiederlein, R. L., Glasser, L. \& Galgiani, J. N. (1987). Bordetella pertussis adenylate cyclase: effects of affinity-purified adenylate cyclase on human polymorphonuclear leukocyte functions. Infection and Immunity 55, 135-140.

Gallin, J. I., Wright, D. G., Malech, H. L., Davis, J. M., KLEMPNER, M. S. \& KIRKPATRICK, C. H. (1980). Disorders of phagocyte chemotaxis. Annals of Internal Medicine 92, 520-538.

Henricks, P. A. J., VAN DeR Tol, M. E., ThysSen, R. M. W. M., VAN Asbeck, B. S. \& Verhoef, J. (1983). Escherichia coli lipopolysaccharides diminish and enhance cell function of human polymorphonuclear leukocytes. Infection and Immunity 41, 294-301.

HewletT, E. L. \& GoRdon, V. M. (1988). Adenylate cyclase toxin of Bordetella pertussis. In Pathogenesis and Immunity in Pertussis, pp. 193-209. Edited by A. C. Wardlaw \& R. Parton. London: John Wiley (in the Press).

Horan, T. D., English, D. \& McPherson, T. A. (1982). Association of neutrophil chemiluminescence with microbicidal activity. Clinical Immunology and Immunopathology 22, 259-269.

Imaizumi, A., Suzuki, Y., ONo, S., Sato, H. \& Sato, Y. (1983). Effect of heptakis(2,6- $O$-dimethyl) $\beta$ cyclodextrin on the production of pertussis toxin by Bordetella pertussis. Infection and Immunity 41, 11381143.

JAMIESON, W. M. (1973). Whooping cough. British Medical Journal 1, 223-225.

LACEY, B. W. (1960). Antigenic modulation of Bordetella pertussis. Journal of Hygiene 58, 57-93.

LACKIE, J. M. (1977). The aggregation of rabbit polymorphonuclear leukocytes (PMN's): effects of agents which affect the acute inflammatory response 
and correlation with secretory activity. Inflammation 2, 1-15.

Lad, P. M., Olson, C. V. \& Smiley, P. A. (1985). Association of the $N$-formyl-Met-Leu-Phe receptor in human neutrophils with a GTP-binding protein sensitive to pertussis toxin. Proceedings of the National Academy of Sciences of the United States of America 82, 869-873.

Manclark, C. R. \& Cowell, J. L. (1984). Pertussis. In Bacterial Vaccines, pp. 69-106. Edited by $\mathbf{R}$. Germanier. London: Academic Press.

Meade, B. D., Kind, P. D., Ewell, J. B., McGrath, P. P. \& MaNClaRK, C. R. (1984). In vitro inhibition of murine macrophage migration by Bordetella pertussis lymphocytosis-promoting factor. Infection and Immunity 45, 718-725.

Nakase, Y., Tateishi, M., Sekiya, K. \& Kasuga, T. (1970). Chemical and biological properties of the purified $\mathrm{O}$ antigen of Bordetella pertussis. Japanese Journal of Microbiology 14, 1-8.

Peppler, M. S. (1984). Two physically and serologically distinct lipopolysaccharide profiles in strains of Bordetella pertussis and their phenotype variants. Infection and Immunity 43, 224-232.

Perera, V. Y., Rogers, H. \& Freer, J. H. (1985). Onedimensional peptide mapping of the subunits of pertussis toxin. Journal of General Microbiology 131, 1897-1901.

Preston, N. W., Tmewell, R. M. \& Carter, E. J. (1980). Experimental pertussis infection in the rabbit: similarities with infection in primates. Journal of Infection 2, 227-235.

Robinson, A., Gorringe, A. R., IRONS, L. I. \& KeEviL, C. W. (1983). Antigenic modulation of Bordetella pertussis in continuous culture. FEMS Microbiology Letters 19, 105-109.

Robinson, A., Duggleby, C. J., Gorringe, A. R. \& LIVEY, I. (1986). Antigenic variation in Bordetella pertussis. In Antigenic Variation in Infectious Diseases, pp. 147-161. Edited by T. H. Birkbeck \& C. W. Penn. Oxford: IRL Press.

Scheffer, J., Konig, W., Hacker, J. \& Goebel, W. (1985). Bacterial adherence and hemolysin production from Escherichia coli induces histamine and leukotriene release from various cells. Infection and Immunity 50, 271-278.

Smith, C. W., Hollers, J. C., Dupree, E., Goldman, A. S. \& LORD, R. A. (1972). A serum inhibitor of leukotaxis in a child with recurrent infections. Journal of Laboratory and Clinical Medicine 79, 878885.

Spangrude, G. J., SACchi, F., Hill, H. R., Van EpPs, D. E. \& DAYNES, R. A. (1985). Inhibition of lymphocyte and neutrophil chemotaxis by pertussis toxin. Journal of Immunology 135, 4135-4143.

Stainer, D. W. \& Scholte, M. J. (1971). A simple chemically defined medium for the production of phase I Bordetella pertussis. Journal of General Microbiology 63, 211-220.

STENDAHL, O. (1983). The physicochemical basis of surface interaction between bacteria and phagocytic cells. In Medical Microbiology 3, Role of the Envelope in the Survival of Bacterial Infection, pp. 137-152. Edited by C. S. F. Easmon, J. Jeljaszewicz, M. R. Brown \& P. A. Lambert. London: Academic Press.

TOPLey, N., Harber, M. J. \& Asscher, A. W. (1984). Influence of bacterial surface components on the stimulation of phagocyte chemiluminescence. In Analytical Applications of Bioluminescence and Chemiluminescence, pp. 409-410. Edited by L. J. Kricka, P. E. Stanley, G. H. Thorpe \& T. P. Whitehead. London: Academic Press.

TuOmanen, E. \& WeISS, A. (1985). Characterization of two adhesins of Bordetella pertussis for human ciliated respiratory-epithelial cells. Journal of Infectious Diseases 152, 118-125.

Wardlaw, A. C. \& Parton, R. (1983). Bordetella pertussis toxins. Pharmacology and Therapeutics 19, 1-53.

Weiss, A. A. \& HewletT, E. L. (1986). Virulence factors of Bordetella pertussis. Annual Review of Microbiology 40, 661-686.

Weiss, A. A., Hewlett, E. L., Myers, G. A. \& FaLkow, S. (1983). Tn5-induced mutations affecting virulence factors of Bordetella pertussis. Infection and Immunity 42, 33-41.

Wilkinson, P. C. (1982). Chemotaxis and Inflammation, 2nd edn. Edinburgh: Churchill-Livingstone. 